\title{
A system of microcalcifications detection and evaluation of the radiologist: comparative study of the three main races in Malaysia
}

\begin{abstract}
This paper uses wavelets in the detection comparison of breast cancer among the three main races in Malaysia: Chinese, Malays, and Indians followed by a system that evaluates the radiologist's findings over a period of time to gauge the radiologist's skills in confirming breast cancer cases. The db4 wavelet has been utilized to detect microcalcifications in mammogram-digitized images obtained from Malaysian women sample. The wavelet filter's detection evaluation was done by visual inspection by an expert radiologist to confirm the detection results of those pixels that corresponded to microcalcifications. Detection was counted if the wavelet-detected pixels corresponded to the radiologist's identified microcalcification pixels. After the radiologist's detection confirmation a new client-server radiologist recording and evaluation system is designed to evaluate the findings of the radiologist over some period of cancer detection working time. It is a system that records the findings of the Malaysian radiologist for the presence of breast cancer in Malaysian patients and provides a way of registering the progress of detecting breast cancer of the radiologist by tracking certain metric values such as the sensitivity, specificity, and receiver operator curve (ROC). The initial findings suggest that no single race mammograms are easier for wavelets' detections of microcalcifications and for the radiologist confirmation even though for this study the Chinese race samples detection average were a few percentages less than the other two races, namely the Malay and Indian races. (C) 2004 Elsevier Ltd. All rights reserved.
\end{abstract}

Keyword: Cancer; Mammogram; Microcalcifications; Radiologist; Wavelets 